\title{
The Jet-Cloud Interaction in 3CR Galaxies: Preliminary Results in Four Galaxies
}

\author{
C. Feinstein ${ }^{1}$, F. D. Macchetto ${ }^{2}$, M. F. Montero ${ }^{3}$, and G. F. Hägele \\ ${ }^{1}$ Facultad de Ciencias Astronómicas y Geofísicas, Paseo del Bosque S/N, 1900, La Plata, \\ Argentina, Ialp, Conicet. email: cfeinstein@fcaglp.unlp.edu.ar \\ ${ }^{2}$ Space Telescope Science Institute, 3700 San Martin Drive, MD21218, Baltimore, USA \\ ${ }^{3}$ Instituto de Astronomía y Física del Espacio, Universidad de Buenos Aires, 1428, Argentina \\ ${ }^{4}$ Dpto. de Física Teórica, C-XI, Universidad Autónoma de Madrid, Ciudad Universitaria de \\ Cantoblanco, 28049, Madrid, Spain. email: guille.hagele@uam.es
}

\begin{abstract}
We present preliminary results of the Gemini/GMOS longslit spectra for four radiogalaxies: 3C 135, 3C 180, 3C 234 and 3C 284. These objects are a subsample of a set of galaxies with noticeable extended structure selected from the HST/WFPC2 3CR Snapshot Survey, data taken with the filters F555W, F702W and narrow-ramp. All of these objects show large regions of [OIII] $\lambda 5007$ emission (narrow ramp filter) and the broad-band filters data show similar structures indicating the presence of strong emission in several lines over these regions. The morphology observed seems to be related (e.g. same position angle, direct overlapping or similar shape) with the radio-jet. For some candidates with these properties GMOS/Gemini spectroscopy was taken. These data (both HST direct imaging and Gemini spectroscopy) can be tested with diagnostic diagrams and total UV photons budget to understand the source of energy that is ionizing the gas. This source of ionization was commonly believed to be the UV photons emitted by the powerful AGN, but several of these objects shows clearly that shocks produce by the radio jet are the main cause of the observed gas line emission.
\end{abstract}

\section{Results}

In the case of 3C 135 the line ratios from GMOS/Gemini longslit spectra, taken in the jet orientation, indicates the presence of high speed shocks in the observed region. This is consistent with the models developed by Dopita et al. (1996 ApJ.455,468), in which a shock plus a precursor HII region could explain the observed line ratio diagnostic. From the [OIII] $\lambda 5007 / \mathrm{H} \beta$ and [OII] $\lambda 3727 /[\mathrm{NeIII}] \lambda 3868$ line ratios we can estimate that a model with $\mathrm{v}=500 \mathrm{~km} \mathrm{sec}^{-1}$ plus a precursor HII region can explain our observations quite well. The $[\mathrm{OII}] \lambda 3727 /[\mathrm{OIII}] \lambda 5007$ ratio, which is very sensible to the parameter $\mathrm{U}$, shows that the observed line ratio does not follow the dilution model of the ionizing radiation filed for a central source of photons $\left(\mathrm{U} \sim \mathrm{r}^{-2}\right)$. Unless there is a large increase of the density of the ISM.

For 3C 180 and 3C 234 the analysis of emission in the longslit spectra shows that shocks with $\mathrm{v}=500 \mathrm{~km} \mathrm{sec}^{-1}$ plus a precursor HII region can explain our observations quite well. In both galaxies the ratio [OII] $\lambda 3727 /[\mathrm{OIII}] \lambda 5007$ is not consistent with photoionization by the AGN.

3C 284, displays two symmetric spots to the NE and SW. The spectra shows that there are three components with different velocities $\left(-500,0\right.$ and $\left.270 \mathrm{~km} \mathrm{sec}^{-1}\right)$ to the SE. From the $[\mathrm{OIII}] \lambda 5007 / \mathrm{H} \beta$ ratio a model with less that $\mathrm{v}=500 \mathrm{~km} \mathrm{sec}^{-1}$ plus a precursor HII region can explain these emission lines, but for the $[\mathrm{OII}] \lambda 3727 /[\mathrm{NeIII}] \lambda 3868$ ratio a higher velocity is needed. 